\title{
Application of UV Imaging in Formulation Development
}

Sun, Yu; Østergaard, Jesper

Published in:

Pharmaceutical Research

DOI:

10.1007/s11095-016-2047-5

Publication date:

2017

Document version

Peer reviewed version

Document license:

CC BY-NC

Citation for published version (APA):

Sun, Y., \& Østergaard, J. (2017). Application of UV Imaging in Formulation Development. Pharmaceutical Research, 34(5), 929-940. https://doi.org/10.1007/s11095-016-2047-5 


\title{
Application of UV Imaging in Formulation Development
}

\author{
Yu Sun and Jesper Østergaard*
}

Department of Pharmacy, Faculty of Health and Medical Sciences, University of Copenhagen, Universitetsparken 2, DK-2100 Copenhagen $\emptyset$, Denmark.

Application of UV Imaging in Formulation Development

(Running head)

* Jesper Østergaard

Department of Pharmacy, Faculty of Health and Medical Sciences, University of Copenhagen, Universitetsparken 2, DK-2100 Copenhagen $\varnothing$, Denmark

Tel.: +45 35336138; fax: +45 35336030.

E-mail address: jesper.ostergaard@ sund.ku.dk

This is a post-peer-review, pre-copyedit version of an article published in Pharmaceutical Research.

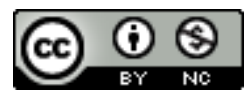

The final authenticated version is available online at:

https://doi.org/10.1007/s11095-016-2047-5 


\begin{abstract}
Efficient drug delivery is dependent on the drug substance dissolving in the body fluids, being released from dosage forms and transported to the site of action. A fundamental understanding of the interplay between the physicochemical properties of the active compound and pharmaceutical excipients defining formulation behavior after exposure to the aqueous environments and pharmaceutical performance is critical in pharmaceutical development, manufacturing and quality control of drugs. UV imaging has been explored as a tool for qualitative and quantitative characterization of drug dissolution and release with the characteristic feature of providing real-time visualization of the solution phase drug transport in the vicinity of the formulation. Events occurring during drug dissolution and release, such as polymer swelling, drug precipitation/recrystallization, or solvent-mediated phase transitions related to the structural properties of the drug substance or formulation can be monitored and characterized by other analytical techniques like Raman spectroscopy. UV imaging is non-intrusive and simple-to-operate, which holds potential for providing a mechanistic foundation for formulation development. This review aims to cover applications of UV imaging in the early and late phase pharmaceutical development with a special focus on the relation between structural properties and performance. Potential areas of future advancement and application are also discussed.
\end{abstract}

\title{
KEY WORDS
}

Diffusion $\cdot$ Dissolution $\cdot$ Drug release $\cdot$ Solid state $\cdot$ UV imaging

\section{ABBREVIATIONS}


ASD: Amorphous solid dispersion

API: Active pharmaceutical ingredient

BCS: Biopharmaceutics classification system

CARS: Coherent anti-Stokes Raman scattering

CBZ: Carbamazepine

CMOS: Complementary metal oxide semiconductor

DVS: Dynamic vapour sorption

FaSSIF: Fasted state simulated intestinal fluid

FTIR: Fourier transform infrared

HPMC: Hydroxypropyl methylcellulose

HP55: Hydroxypropyl methylcellulose phthalate

MRI: Magnetic resonance imaging

NIR: Near-infrared

PLLA: Poly-L-lactic acid

PC: Phosphatidylcholine

NIC: nicotinamide

SLS: Sodium lauryl sulfate

SEM: Scanning electron microscopy

UV: Ultraviolet

Vis: Visible

XRPD: X-ray powder diffraction 


\section{INTRODUCTION}

In vitro dissolution and release testing is a key activity in the pharmaceutical industry throughout the various phases of drug development. For example, the dissolution behavior of candidate molecules is assessed in early phase drug development as part of solid form selection; dissolution testing is used to guide dosage form development, in vitro - in vivo correlations and assessment of batch-to-batch quality [1-10]. The compendial dissolution testing methods have been criticized for variability and lack of biorelevance but remain essential tools for asserting performance and quality of drug products [4-6]. The compendial methods require large volumes of dissolution medium and sample which could not be suitablein early phase drug development due to only limited quantities of drug substance available. To this end, miniaturized or micro-dissolution apparatuses have been developed to overcome this chanllege and might potentially become a useful tool in pharmaceutical industry for drug candidate screening [7-9]. A number of the commonly employed dissolution and release testing methods rely on bulk solution measurements for drug quantification whereby dissolution events such as polymer swelling, drug precipitation, or solvent-mediated solid form transformation might not be detected [11-16]. Hence, platforms capable of providing real-time mechanistic information related to drug dissolution and release processes are desirable for an indepth understanding of these complex events.

Imaging techniques providing spatially, and temporally resolved information on drug delivery systems are increasingly used in formulation design and development. These techniques include Raman spectroscopic methods [17-19], magnetic resonance imaging (MRI) [20-24], Fourier transform infrared (FTIR) spectroscopic imaging [25-27], near-infrared (NIR) spectroscopy and imaging [28,29], coherent anti-Stokes Raman scattering (CARS) [30,31] and UV imaging [32]. Information related to for example, water penetration, polymer swelling and dissolving, gel layer formation, drug concentration mapping inside and outside the formulation can be gained through 
those versatile imaging platforms, e.g., [10,20,25,28,29,32,33]. However, for the purpose of dissolution and release testing, some of the imaging techniques are not fully commercially developedor well-established. , [10]. Ultraviolet-visible (UV-Vis) spectrophotometry has been used for decades for quantification due to the fact that most active pharmaceutical ingredients contain chromophores that absorb light in the UV or visible range [34]. UV imaging was introduced for drug release testing in 2010 [35] and is still a relatively new technology in formulation research. The creation of real-time images of API transport in or in the close proximity of the solid or the formulation subject to investigation have spurred interest in various areas from the determination of intrinsic dissolution rates to characterization of the in vitro performance of drug delivery systems such as studies on drug release kinetics or polymer swelling behavior. Relative to other imaging techniques, the non-intrusive measurements by UV-Vis imaging of the solution phase provide a complementary and comparatively simple platform for in vitro drug dissolution and release studies.

The aim of this review is to discuss and assess the potential use of UV-Vis imaging in relation to formulation development. The UV imaging instrumentation and associated advantages and limitations have been covered in detail previously [32]. Thus, the current review aims to cover recent applications of UV-Vis imaging in the early and late phase drug development. Particular focus will be directed to the cases where UV imaging has been found useful for elucidating the interplay between structure and drug release.

\section{UV IMAGING INSTRUMENTATION}

UV imaging utilizes the native characteristic UV absorbance to create spatially and temporally resolved analyte concentration maps within a transparent material. The commercially available Sirius SDI (surface dissolution imaging) UV imaging system utilizes a pulsed Xe lamp as the light source, incorporates a band pass filter for wavelength selection, a fibre optic cable for presenting 
the light to sample quartz cell, a lens for collimating the light and a CMOS (complementary metal oxide semiconductor) camera chip measuring the light intensity. The nominal imaging area of the Sirius SDI is $7 \times 9 \mathrm{~mm}^{2}$ composed of $1024 \times 1280$ pixels each having the size of $7 \times 7 \mu \mathrm{m}^{2}$. The CMOS chip is only active in the visible wavelength range and a phosphorous coating in front of the chip converts the UV light into the visible range prior to detection [36]. The instrument design allows the use of only one wavelength at a time. For a detailed treatment of the performance characteristics of the SDI UV imaging instrument (and the related D100 unit), readers are referred to [36]. The UV imaging, or rather UV-Vis, imaging systems were tested in the wavelength interval 200 to $650 \mathrm{~nm}$. Due to the special configuration of light source, phosphorous conversion and CMOS chip, the SDI system appears to have a "blind" wavelength range from 300 to $350 \mathrm{~nm}$ where it is difficult or impossible to obtain good measurements due to lack of detector response. The resolution of the instrument was found to be in the range of $\sim 50$ to $200 \mu \mathrm{m}$ depending on configuration and sample alignment [36]. This should be kept in mind when considering work with nano- and micro-particulates. Typically the light source and camera unit is attached to a syringe pump, temperature control unit and a low-volume flow-through quartz cell as shown schematically in Fig. 1. However, the design of the camera housing is compatible with various types of sample holders and quartz cells. Schematic representations of quartz cells applied for dissolution and release testing are shown in Fig. 2.

\section{UV IMAGING IN EARLY DRUG DEVELOPMENT}

Preformulation studies are an essential component in drug development to gain fundamental knowledge about the physicochemical properties of drug candidates in order to guide the development of an optimal pharmaceutical formulation. In the past few decades, an increasing number of new potential drug molecules which exhibite reasonable membrane permeability but 
poor aqueous solubility have been discovered $[4,5,37,38]$. Hence, dissolution will often be the ratelimiting step for in vivo drug absorption. Solid-form screening and selection aiming to identify the optimal solid form of the drug candidate as early as possible is an additional important part of the preformulation activities. The different solid forms of an API, potential solvent-mediated phase transformations, the presence of ions, surfactants or biorelevant components in the dissolution medium might significantly affect dissolution behaviour, which necessitates an in-depth understanding. UV imaging has been found useful in this area. An initial report [39] applied UV imaging with the assistance of Raman spectroscopy to assess the dissolution and identify the solid forms before and after dissolution of amlodipine free base, amlodipine besylate dihydrate and amorphous amlodipine besylate using the flow-through dissolution setup shown in Fig. 1. UV imaging possessed the discriminatory power to rank the three solid forms in terms of dissolution rate. Raman spectroscopy confirmed solid from changes inferred from the dissolution rate versus time profiles [39]. In a similar type of study, UV imaging was successfully employed to determine the dissolution properties of a range of compounds and solid forms, namely: theophylline (BSC I, anhydrate and monohydrate), indomethacin (BCS II, alpha and gamma form) and ibuprofen (BCS II, free acid and sodium salt), which, according to the authors, had proved difficult using conventional dissolution testing [40]. For example, the conversion of anhydrous theophylline to monohydrous theophylline in aqueous environments consititutes the main challenge for dissolution studies of theophylline anhydrate. However, due to fast analysis time and different hydrodynamic condition in UV imaging flow-through setup, this solid from transformation was not observed during the experiments and UV imaging demonstrated high discriminatory power [40]. Pharmaceutical cocrystals have emerged as an approach for improving solubility and bioavailability of poorly soluble drugs [41-44]. Li and coworkers investigated the dissolution and transformation behavior of carbamazepine-nicotinamide (CBZ-NIC) cocrystals [45] as well as the effect of using surfactant- 
containing dissolution media on CBZ-NIC solubility and dissolution behavior using UV imaging, Raman spectroscopy and SEM [46]. The dissolution rate of CBZ-NIC cocrystals was higher within the first $3 \mathrm{~h}$ as compared to the carbamazepine form I and III as well as the dihydrate [45]. Off-line Raman spectroscopy revealed that CBZ-NIC cocrystal, carbamazepine I, carbamazepine III, and physical mixture transformed into the stable carbamazepine dihydrate during dissolution [45]. In the following study, the surfactants sodium lauryl sulfate (SLS) and Tween 80 had an interesting effect on the dissolution behavior of the CBZ-NIC cocrystal [46]. SLS and Tween 80 had little influence on the solubility of the cocrystal, but they had opposite effects on the intrinsic dissolution rate of the CBZ-NIC cocrystal. SLS increased the intrinsic dissolution rate of the CBZ-NIC cocrystal whereas Tween 80 apparently decreased its intrinsic dissolution rate probably related to the differences in their molecular structure and solubilisation mechanism [46]. For the CBZ-NIC cocrystal it was possible to find at suitable detection wavelength, however, multicomponent systems may pose a challenge using current UV imaging instrumentation where only one wavelength is available at a time.

Application of physiologically relevant dissolution media in dissolution testing is important in order to simulate the in vivo situation as closely as possible and, also, UV imaging have been conducted using bio-relevant media [47-49]. Gordon et al. found that the dissolution behavior of furosemide was not significantly influenced by applying the fasted state simulated intestinal fluid as compared to pure buffer solution [47]. For a number of samples, pronounced irregular upward shifts of the contour lines in UV absorbance maps were observed. Such observations may indicate dissolution followed by precipitation associated with solid form transformation or swelling of the solid material. In the particular case, in situ Raman spectroscopy as well as XRPD analysis demonstrated that the observed swelling at the compact surface was not associated with a solid form transformation [47]. Such an occurrence remains an observation of interest. Subsequently, the 
dissolution behavior of amorphous furosemide sodium salt and amorphous furosemide (acidic neutral species) in simulated bio-relevant intestinal medium was studied in comparison with the corresponding crystalline forms [48]. The two furosemide sodium salt forms (amorphous or crystalline) were found to have higher dissolution rates than the two acidic forms. The conversion of the amorphous form to crystalline form was fast in both cases as detected by Raman spectroscopy. Niederquell and Kuentz applied UV imaging-based dissolution testing to the poorly water soluble acids celecoxib, ketoprofen, naproxen, and sulfathiazole in fasted state simulated intestinal fluid [49]. For all four compounds, a high and variable initial dissolution rate was followed by decreasing dissolution rates with time approaching a pseudo-equilibrium state. This time dependent dissolution behaviour, which may be found in other UV imaging studies as well, was related to sample heterogeneiety and discussed in terms of fractal-like dissolution behavior [49]. This important study emphasizes the importance of studying heterogeneity-related effects in small scale in vitro dissolution testing.

Identification of solid form transformations in relation to the in vitro dissolution studies has mainly been achieved using off-line Raman spectroscopy or XRPD, normally performed before and after dissolution measurements. Alternatively, as reported by Nielsen et al. [48], in situ Raman spectroscopy can be performed using a flow-through cell similar to that used with the UV imaging system making essentially parallel studies. However, in cases where the API availability is limited, parallel measurements would not be feasible and off-line studies may be unlikely to capture the transformation kinetics due to changes in hydrodynamic conditions. Østergaard et al. developed an integrated dissolution setup combining UV imaging with a Raman spectroscopy probe [50]. The dissolution of sodium naproxenate in dilute hydrochloric acid and theophylline anhydrate in water constituted the model systems applied for demonstrating the suitability of the approach. Conversion of theophylline anhydrate into the monohydrate was observed as expected from literature. More 
interestingly, two intermediate forms were detected by Raman spectroscopy during the transformation of sodium naproxenate to the neutral naproxen form upon dissolution in $0.1 \mathrm{M} \mathrm{HCl}$ [50]. Such intermediate forms might otherwise be overlooked through traditional dissolution method or an offline Raman spectroscopic characterization.

The UV imaging-based dissolution studies performed using the flow-through setup highlight the significance of real-time information provided by UV imaging as well as the importance of combining with suitable solid-state characterization techniques. Key features of the so-called surface dissolution imaging setup include low sample requirements (3 to $10 \mathrm{mg}$ ), potential for rapid dissolution tests, testing periods from $10 \mathrm{~min}$ to several hours, possibility to apply bio-relevant dissolution media (this may be wavelength specific), and information related to the behavior of the solid material upon exposure to the dissolution medium. The imaging offers important qualitative information regarding the dissolution process in addition to the typical quantitative data. In the hands of the authors, flow-through dissolution testing using UV imaging tend to be less repeatable/robust as compared to what is expected from traditional dissolution tests, this may be related to the small scale and sample preparation issues. As an example, Boetker et al. employed finite element simulation to characterize the hydrodynamics of the UV imaging flow cell [51]. Good agreement was found between the dissolution rates determined from the collected effluents and the developed numerical model. However, deviations were observed upon comparison of the dissolution rates determined from the UV images using the instrument software to those obtained from the finite element simulations and the effluents. Suggested causes for the discrepancy related to the finite size of the pixels, that the incident light had an angle different from $0^{\circ}$, and formation of the density gradients around the compact surface [51]. Since the time of the study, a lens for improving collimation of the incident light has been incorporated. In any case, further 
characterization of the UV imaging system may be needed to clarify the role of these factors on dissolution rate determination.

Of particular interest in a preformulation setting, $\mathrm{Lu}$ and $\mathrm{Li}$ recently reported on a UV imaging method for simultaneous determination of drug solubility and diffusion coefficient by fitting imaging data to a numerical model derived from Fick's second law of diffusion [52]. The solubilities calculated for carbamazepine and ibuprofen were in good agreement with literature data. Experiments were executed within $5 \mathrm{~min}$, allowing estimation of the apparent solubility of a thermodynamically unstable compunds, e.g., carbamazepine form II [52].

Imaging the dissolution of individual crystals may be of interest since it might provide fundamental knowledge regarding the dissolution mechanism of a crystalline API. A first attempt of applying UV imaging in this area investigated the dissolution of a single crystal of lidocaine in stagnant buffer solution [53]. The absorbance maps clearly visualized the influence of natural convection due to density gradients on the dissolution of lidocaine. It is tempting to speculate that such measurements can be further developed into a setup where face-specific dissolution can be investigated.

Assessment of micro-environmental $\mathrm{pH}$ changes during dissolution may be of relevance for ionizable drug substances as well as their formulations since the local $\mathrm{pH}$ influences dissolution and release performance [54]. A proof of concept study mapped the local $\mathrm{pH}$ change in relation to the dissolution of sodium naproxenate in $0.01 \mathrm{M}$ and $0.10 \mathrm{M}$ hydrochloric acid using a Vis imagingbased approach and a $\mathrm{pH}$ indicating dye thymol blue [55]. The approach should be termed Vis imaging rather than $\mathrm{UV}$ imaging since the local $\mathrm{pH}$ was assessed from the change in absorbance at $550 \mathrm{~nm}$. Results showed the dissolution of sodium naproxenate could cause significant temporary $\mathrm{pH}$ changes in the dissolution medium in the vicinity of the solid salt. Parallel UV imaging studies 
monitored the dissolution of sodium naproxenate under the conditions where the salt was eventually neutralized and precipitated as the acidic species naproxen.

To sum up, UV imaging in combination with solid state characterization techniques such as Raman spectroscopy seems to offer an interesting experimental setup in preformulation and early phase development facilitating basic dissolution behavior to be monitored. A caveat may be that studies have been performed using well-known model APIs. Cases where UV imaging data have been succesfull (or the opposite) in progressing development candidates are less likely to become public available.

\section{UV IMAGING IN FORMULATION DEVELOPMENT}

Much effort is put into delineating the interdependence between dosage form structure and release kinetics. Imaging techniques can be used to visualize, for instance, how drug-polymer interactions affect release upon exposure to aqueous environment. In general, as compared to other chemical imaging techniques, UV images contain little structural information image. .Still, as will be discussed in the following, UV imaging is likely to play a role in the characterization of drugexcipient interactions and formulation development. This section covers applications of UV imaging in this area divided according to route of administration.

\section{Oral Route of Administration}

Hydrophilic polymers are widely used to control the release rate of API in tablets for oral administration. Visualization of the swelling process of hydroxypropyl methylcellulose (HPMC) in an aqueous solution has been achieved by MRI [22,56,57], FTIR imaging [58-61] or near infrared chemical imaging [29]. Pajander et al. investigated HPMC swelling using UV imaging under stagnant conditions as well as in the presence of flow [62]. Compacts of two viscosity grades of 
HPMC, $15 \mathrm{cP}$ and $50 \mathrm{cP}$, were prepared and subjected to real-time visualization of the polymer behavior at $214 \mathrm{~nm}$. It is widely accepted that several events take place sequentially upon HPMC contact with aqueous solution $[63,64]$. The polymer entanglement and swelling leading to the formation of a gel layer was apparent by UV imaging. Distinct features such as the diffusion layer, erosion front and gel point was inferred from the images. The swelling behavior of the $15 \mathrm{cP}$ and 50 cP HPMC qualities was slightly different as characterized by UV imaging, the higher viscosity grade HPMC swelled faster and formed a thicker gel layer, being less susceptible to erosion in the presence of flow [62].

Amorphous solid dispersions (ASDs) constitute a potential avenue for improving oral bioavailability. Amorphous solid dispersions involve amorphization of a crystalline API by dispersion in an amorphous hydrophilic polymer with a high glass transition temperature (Tg) [6567]. The molecular mobility of the API is significantly reduced and stabilized against crystallization while embedded in the polymer matrix [68]. A key hurdle associated with the development of ASDs may be a limited understanding of the relationship between physical structure, stability and pharmaceutical performance $[66,68]$. Nilotinib exhibited improved bioavailability in dogs when formulated with hydroxypropyl methylcellulose phthalate (HP55) and surfactants upon carbondioxide-mediated processing [69]. Colombo et al. systemically studied the polymer matrix effects on dissolution and re-precipitation kinetics mediated by this ASD formulation using simultaneous UV-imaging and Raman spectroscopy [70]. HP55, crystalline nilotinib, HP55-nilotinib ASDs with $20 \%$ and $40 \%$ drug load and the corresponding physical mixture were investigated in a simulated gastrointestinal environment. The structural changes with respect to swelling and dissolution behavior of the polymer in an acidic environment $(\mathrm{pH} 2.2)$ as well as neutral solution ( $\mathrm{pH}$ 6.4) were reflected as changes in matrix height (swelling) at the center of the compacts (Fig. 3). Results showed that different drug loads led to distinctively different formulation behaviour. A $20 \%$ drug 
load suppressed the polymer swelling and seemingly formed a homogenous gel layer in acidic medium. After a change to near neutral $\mathrm{pH}$, the dissolution of the polymer did not occur immediately as for pure HP55 compacts; instead, a slight increase in matrix height was observed which might suggest a re-organization of the gel structure induced by the $\mathrm{pH}$ change. High nilotinib load (40\%) prevented matrix swelling even further at acidic $\mathrm{pH}$ and remained stable in neutral environment. With respect to dissolution, the $40 \%$ drug load formulation displayed a remarkable increased dissolution after change into the neutral $\mathrm{pH}$ as compared to the other samples. Those findings are of interest since utilizing HPMC with the absence of phthalate groups did not show drug-load dependent swelling and dissolution behavior. These results in combination with the data obtained from simultaneous Raman spectroscopy demonstrated that higher drug load formulation (40\% nilotinib) efficiently prevented the recrystallization of nilotinib from the amorphous solid dispersion after being exposed to the $\mathrm{pH} 6.4$ medium. The drug-polymer hydrogen-bonding, mediated by the phthalate groups was crucial in determining this effect. This study highlights the potential application of UV imaging in revealing the interplay of dosage form microstructure and dosage form performance.

Lipid-based drug delivery systems constitute a versatile approach for increasing the bioavailability of poorly soluble APIs $[38,71]$. Gautschi et al. studied drug release from drugphospholipid complexes by UV imaging [72]. Formulations were prepared using monoacyl or diacyl phosphatidylcholine (PC) as excipients and a series of poorly soluble compounds (BCS II and IV) to characterize the feasibility of forming drug-phospholipid dispersions at a 1:1 molar ratio. The ability to form solid dispersions with the phospholipids was related to API physical chemical parameters such as frontier orbital energy, enthalpy of fusion, and $\log$ P [72]. Dissolution studies using UV imaging showed a remarkable surface swelling of the solid dispersions over the compact surface due to phospholipid hydration. Monoacyl PC enhanced drug release rate, whereas diacyl PC 
had only limited effect on the drug dissolution rates. Two factors were suggested to contribute to this observation, drug precipitation during hydration and the inability to form micelles in case of the diacyl PC as compared to mono-acyl PC [72].

Gaunø et al. applied UV imaging to monitor the in vitro release behavior of single 5aminosalicylic acid extrudates with different thicknesses of ethyl cellulose coating applied and compared the results with traditional bulk release testing using the traditional paddle method [73]. The individual extrudates $(\sim 1 \mathrm{~mm}$ in length) were embedded in an agarose hydrogel matrix keeping the extrudate in position during the measurements. Both UV imaging and paddle dissolution method confirmed that the release of 5-aminosalicylic acid from the extrudates decreased with increasing film coating layer thickness [73]. UV imaging was also capable of visualizing coating defects inflicted on the extrudates [73]. As a proof of concept study, the extrudate was manually damaged at different area and 5-aminosalicylic acid was found released faster from the compromised area leading to the formation of an asymmetrical absorbance map around the extrudate in comparison with the symmetrical spherical absorbance maps formed around the undamaged extrudates [73]. UV imaging measurements might provide a rapid and simple platform for detection of film coating defects [73].

Drug nano-crystals are investigated as an approach to increase the biavailability of poorly soluble drugs [74-76]. Indomethacin nano-crystals with different particle sizes in the range of 580-950 nm were prepared using poloxamer F68, poloxamer F127 and polysorbate 80 as stabilizers through a top-down wet milling technique [77]. The dissolution of indomethacin from compacts formed from compression of the nano-crystals was compared to the dissolution of unmilled indomethacin as well as the corresponding physical mixture using UV imaging and a traditional flow-through dissolution method. Initial UV and Vis imaging experiments revealed a significant increase in absorbance 
during flow-through dissolution at $265 \mathrm{~nm}$ where indomethaxin absorbs light strongly as well as at $550 \mathrm{~nm}$ where indomethacin has no absorbance. This observation was ascribed to the nanocrystals escaping the compact surface to the dissolution medium leading to the light scattering instead of absorbance due to molecularly dissolved indomethacin [77]. The dissociation of particles from the surface was suppressed using a hydrogel matrix. Under these conditions, the nano-crystal compacts exhibited a significantly higher dissolution rate as compared to unmilled indomethacin [77]. The indomethacin concentration in the immediate vicinity of the nano-crystal compact was almost five times the solubility which might be related to the supersaturation. Although, the solubility enhancing effects by poloxamer F68 or F127 may also contribute to the increased local indomethacin concentration [77].

Micro-fabricated devices have been proposed as novel oral delivery systems since the size and shape of the devices can be manipulated in order to maximize the contact area with absorptive tissue and improve bioavailability [78,79]. Nielsen et al. employed UV imaging as well as the $\mu$ Diss profiler to characterize the release of amorphous sodium salt of furosemide from microwells (240 $\mu \mathrm{m}$ inner diameter) fabricated with poly-L-lactic acid (PLLA) and coated with Eudragit® L100 [80]. Simulated gastric and intestinal media were utilized in the dissolution study to be as close as possible to the in vivo situation. Both UV imaging and the $\mu$-Diss profiler revealed the same trend with an absence of drug release in the gastric medium and an immediate furosemide release from the assemblies of coated microwells subject to testing after switching to the simulated intestinal medium due to the $\mathrm{pH}$ change. In this particular study, the UV images were used in a qualitative manner to assess the Eudragit ${ }^{\circledR}$ coating protecting the drug from being released in an acidic environment.

\section{Parenteral Adminstration}


Most approved protein and peptide-based medicines are administered via the parenteral route (intramuscular, subcutaneous or intravenous injection) due to their susceptibility to enzymatic degradation and poor membrane passage [81-83]. However, currently there are no standardized regulatory approved in vitro release testing methods for parenterals $[84,85]$. In vitro platforms capable of predicting bio-performance of parenteral depots for administration into soft tissue are desirable in formulation development. UV imaging-based approaches incorporating a hydrogel matrix as soft tissue model has been proposed for characterization of such depot systems [86-92]. Model drug substances, with low and high molecular weight have been studied to assess the feasibility of this setup in in vitro drug release measurements. Ye et al. employed pluronic F127 as the hydrogel matrix and investigated the diffusion of piroxicam and human serum albumin in the gel matrix using a rectangular parallel piped quartz cell, half of which was filled the formulation, the other half with dissolution medium (see Fig. 2B and 2C) [86]. By fitting the experimental data to an equation derived from Fick's second law, the apparent diffusion coefficients of piroxciam and human serum albumin in 20\% (w/w) F127 were determined. Diffusion and partition of piroxicam from oil solution into hydrogel matrixes (agarose and pluronic F127-based) mimicking subcutaneous tissue were also investigated [87]. In a subsequent study, two piroxicam solutions (aqueous and oily) were injected into an agarose hydrogel matrix using the setup sketched in Fig. 2D in order to more closely simulate subcutaneous administration [88]. Spatial concentration distributions of drug around the injection site were monitored in real-time. From the spatially and temporally resolved absorbance data, diffusion coefficients and distribution coefficients have been obtained using Fick's second law $[87,88]$. Also in the context of subcutaneous administration, Jensen et al. investigated the diffusion of human insulin in an agarose gel matrix [90]. The selfassociation properties of human insulin were reflected in the diffusional transport, essentially determining the hydrodynamic radii corresponding to the insulin monomer, dimer and hexamer 
from the diffusion measurements conducted by UV imaging [90]. Changes in $\mathrm{pH}$ of the surrounding microenvironment during dissolution of amorphous insulin in agarose matrixes at $\mathrm{pH} 3.0,5.4$ and 7.4 [91] were investigated using the $\mathrm{pH}$ indicator method previously introduced [55]. Local $\mathrm{pH}$ changes reflecting protonation and deprotonation of dissolved insulin molecules were proposed to explain anomalous insulin dissolution profiles. In the same context, using human insulin as a model protein, three different in vitro dissolution methods for assessing insulin release from lipid implants were evaluated including: i) agitated vials filled with bulk dissolution medium, ii) release in an agarose gel, insulin quantified in cored-out gel samples (as described in [93]) and iii) in an agarose gel as shown in Fig. 2E with insulin detection via UV imaging [92]. In accordance with expectations, insulin release from Sterotex implants with a drug load of $20 \%$ (w/w) was faster as compared to the release from implants with a drug load of $10 \%$ (w/w). However, interestingly implants with a high drug load of $20 \%$ displayed similar release profiles independent of the in vitro release methods used whereas the implants with a drug load of $10 \%$ exhibited a faster insulin release in an agitated buffer solution than in the stagnant agarose hydrogel matrixes [92]. Further studies are needed to unravel the interplay between release behavior and in vitro release testing conditions. This study highlights the importance of the in vitro testing conditions on drug release from parenteral depot systems and provides proof-of-concept to the use of UV imaging in the characterization of implants. It is also clear that future work could benefit significantly from access to instrumentation with a larger imaging area.

In our view the studies above suggest that hydrogel matrixes are worth pursuing in the search for suitable in vitro release testing methodologies for injectables. For liquid injectables a matrix acts to confine the preformulation or formulation. In combination with UV imaging, transpancy of the matrix is a fundamental requirement but much can be done in terms of altering the properties of the matrix as recently shown. Composite hydrogels containing agarose in combination with the 
biorelevant polyelectrolytes chondroitin sulfate and hyaluronic acid was prepared and the diffusion of low molecular weight substances and two proteins was investigated by UV imaging [89]. Incorporation of the negatively charged polyelectrolytes into the hydrogels reduced the apparent diffusivities of the cationic model compounds as compared to the pure agarose gel. Inclusion of tissue components may be an approach to increase the biorelevance of the in vitro methods as well as to probe the importance of specific interactions for a given API or formulation.

\section{Other Routes of Administration}

The release of nicotine from $2 \mathrm{~mm}$ in diameter samples of Nicorette ${ }^{\circledR}$ transdermal patches into phosphate buffer, $\mathrm{pH}$ 7.40, constituted the first application of UV imaging [35]. Using a flowthrough cell, the release of nicotine into a stagnant or a constant flow of phosphate buffer was monitored in real time. Visualization of the development of nicotine concentration gradients in the aqueous phase next to the sample patch as well as quantification of the nicotine release rate was achieved with UV imaging. The nicotine release rate determined by UV imaging was higher than that attained using the traditional paddle over disk method. This apparent discrepancy was ascribed to edge effects associated with the geometry and positioning of the samples in the flow cell [35].

The antibiotic chloramphenicol was applied as a model drug in the preparation of in situ forming gels intended for ophthalmic administration [94]. The effect of poloxamer concentration, addition of thickener, simulated tear fluid and $\mathrm{pH}$ on the gelation temperature and release rate of chloramphenicol from in situ forming gels was evaluated using a UV imaging based flow-through setup. UV imaging was found suitable for characterization of ophthalmic in situ forming gels being capable of discriminating chloramphenicol release behavior from different formulation compositions [94]. 


\section{CONCLUSION}

With the appearance of new challenging drug molecules, there is a push to develop novel, flexible dissolution testing methods in order to reduce development cost and use less animal experiments. The conventional dissolution testing approaches exhibit a various drawbacks limiting the ability to predict in vivo behavior. Being able to non-invasively image the dissolution process, UV imaging in combination with other spectroscopic techniques such as Raman spectroscopy allows the microenvironment and structure of dosage forms to be characterized with respect to monitoring local $\mathrm{pH}$ changes, polymer swelling, drug precipitation or re-crystallizion, solvent-mediated phase transition, peptide self-association and electrostatic interactions. In comparison to other imaging techniques, like Raman spectroscopy imaging, MRI or Fourier transform infrared (FTIR) imaging, the utilization of UV imaging to study pharmaceutical performances in vitro is still at an ealy stage. The application of UV imaging over the past five years demonstrated its potential use in relation to a range of different drug substances and delivery systems. Considering continuing improvements in hardware, implementation of quality-by-design concepts and benefits of real-time release information, it is anticipated that UV imaging may increasingly used in formulation development.

\section{ACKNOWLEDGEMENTS AND DISCLOSURES}

This project has received funding from the European Union's Horizon 2020 research and innovation programme under the Marie Sklodowska-Curie grant agreement No 644056 (CRYDIS). $\mathrm{J} \varnothing$ is member of the Scientific Advisory Board of Paraytec Ltd (York, UK). The authors alone are responsible for the content and writing of this paper

\section{References}

1. Dokoumetzidis A, Macheras P. A century of dissolution research: From Noyes and Whitney to the Biopharmaceutics Classification System. Int. J. Pharm. 2006;321:1-11. 
2. Morissette S. High-throughput crystallization: polymorphs, salts, co-crystals and solvates of pharmaceutical solids. Adv. Drug Deliv. Rev. 2004;56:275-300.

3. Ekarat Jantratid, Maria Vertzoni. Dissolution Testing to Forecast In Vivo Performance of Immediate-Release Formulations. Oral Drug Absorpt. CRC Press; 2010; p. 224-43.

4. Azarmi S, Roa W, Löbenberg R. Current perspectives in dissolution testing of conventional and novel dosage forms. Int. J. Pharm. 2007;328:12-21.

5. Phillips DJ, Pygall SR, Cooper VB, Mann JC. Overcoming sink limitations in dissolution testing: a review of traditional methods and the potential utility of biphasic systems: Dissolution testing: biphasic systems. J. Pharm. Pharmacol. 2012;64:1549-59.

6. Gray V, Kelly G, Xia M, Butler C, Thomas S, Mayock S. The science of USP 1 and 2 dissolution: present challenges and future relevance. Pharm. Res. 2009;26:1289-302.

7. Ahnfelt E, Sjögren E, Axén N, Lennernäs H. A miniaturized in vitro release method for investigating drug-release mechanisms. Int. J. Pharm. 2015;486:339-49.

8. Tseng Y-C, Patel M, Zhao Y. Determination of Intrinsic Dissolution Rate Using Miniaturized Rotating and Stationary Disk Systems. Dissolution Technol. 2014;21:24-9.

9. Alsenz J, Haenel E, Anedda A, Du Castel P, Cirelli G. Miniaturized INtrinsic DISsolution Screening (MINDISS) assay for preformulation. Ind. Pharm. Sci. 2016;87:3-13.

10. Kuentz M. Analytical technologies for real-time drug dissolution and precipitation testing on a small scale: Real-time analytics for mini dissolution. J. Pharm. Pharmacol. 2015;67:143-59.

11. Lehto P, Aaltonen J, Niemelä P, Rantanen J, Hirvonen J, Tanninen VP, et al. Simultaneous measurement of liquid-phase and solid-phase transformation kinetics in rotating disc and channel flow cell dissolution devices. Int. J. Pharm. 2008;363:66-72.

12. Savolainen M, Kogermann K, Heinz A, Aaltonen J, Peltonen L, Strachan C, et al. Better understanding of dissolution behaviour of amorphous drugs by in situ solid-state analysis using Raman spectroscopy. Eur. J. Pharm. Biopharm. 2009;71:71-9.

13. Allesø M, Chieng N, Rehder S, Rantanen J, Rades T, Aaltonen J. Enhanced dissolution rate and synchronized release of drugs in binary systems through formulation: Amorphous naproxencimetidine mixtures prepared by mechanical activation. J. Controlled Release. 2009;136:45-53.

14. Boetker JP, Rantanen J, Arnfast L, Doreth M, Raijada D, Loebmann K, et al. Anhydrate to hydrate solid-state transformations of carbamazepine and nitrofurantoin in biorelevant media studied in situ using time-resolved synchrotron X-ray diffraction. Eur. J. Pharm. Biopharm. 2016;100:119-27.

15. Thiry J, Broze G, Pestieau A, Tatton AS, Baumans F, Damblon C, et al. Investigation of a suitable in vitro dissolution test for itraconazole-based solid dispersions. Eur. J. Pharm. Sci. 2016;85:94-105. 
16. Punčochová K, Vukosavljevic B, Hanuš J, Beránek J, Windbergs M, Štěpánek F. Non-invasive insight into the release mechanisms of a poorly soluble drug from amorphous solid dispersions by confocal Raman microscopy. Eur. J. Pharm. Biopharm. 2016;101:119-25.

17. Tres F, Patient JD, Williams PM, Treacher K, Booth J, Hughes LP, et al. Monitoring the Dissolution Mechanisms of Amorphous Bicalutamide Solid Dispersions via Real-Time Raman Mapping. Mol. Pharm. 2015;12:1512-22.

18. Paudel A, Raijada D, Rantanen J. Raman spectroscopy in pharmaceutical product design. Adv. Drug Deliv. Rev. 2015;89:3-20.

19. Smith GP, McGoverin CM, Fraser SJ, Gordon KC. Raman imaging of drug delivery systems. Adv. Drug Deliv. Rev. 2015;89:21-41.

20. Richardson J, Bowtell R, Mader K, Melia C. Pharmaceutical applications of magnetic resonance imaging (MRI). Adv. Drug Deliv. Rev. 2005;57:1191-209.

21. Tres F, Coombes S, Phillips A, Hughes L, Wren S, Aylott J, et al. Investigating the Dissolution Performance of Amorphous Solid Dispersions Using Magnetic Resonance Imaging and Proton NMR. Molecules. 2015;20:16404-18.

22. Gajdošová M, Pěček D, Sarvašová N, Grof Z, Štěpánek F. Effect of hydrophobic inclusions on polymer swelling kinetics studied by magnetic resonance imaging. Int. J. Pharm. 2016;500:136-43.

23. De Smet M, Heijman E, Langereis S, Hijnen NM, Grüll H. Magnetic resonance imaging of high intensity focused ultrasound mediated drug delivery from temperature-sensitive liposomes: An in vivo proof-of-concept study. J. Controlled Release. 2011;150:102-10.

24. Kempe S, Metz H, Pereira PGC, Mäder K. Non-invasive in vivo evaluation of in situ forming PLGA implants by benchtop magnetic resonance imaging (BT-MRI) and EPR spectroscopy. Eur. J. Pharm. Biopharm. 2010;74:102-8.

25. Kazarian SG, Ewing AV. Applications of Fourier transform infrared spectroscopic imaging to tablet dissolution and drug release. Expert Opin. Drug Deliv. 2013;10:1207-21.

26. Pudlas M, Kyeremateng SO, Williams LAM, Kimber JA, van Lishaut H, Kazarian SG, et al. Analyzing the impact of different excipients on drug release behavior in hot-melt extrusion formulations using FTIR spectroscopic imaging. Eur. J. Pharm. Sci. 2015;67:21-31.

27. Ewing AV, Biggart GD, Hale CR, Clarke GS, Kazarian SG. Comparison of pharmaceutical formulations: ATR-FTIR spectroscopic imaging to study drug-carrier interactions. Int. J. Pharm. 2015;495:112-21.

28. Reich G. Near-infrared spectroscopy and imaging: Basic principles and pharmaceutical applications. Adv. Drug Deliv. Rev. 2005;57:1109-43.

29. Li W, Woldu A, Araba L, Winstead D. Determination of Water Penetration and Drug Concentration Profiles in HPMC-Based Matrix Tablets by Near Infrared Chemical Imaging. J. Pharm. Sci. 2010;99:3081-8. 
30. Fussell A, Garbacik E, Offerhaus H, Kleinebudde P, Strachan C. In situ dissolution analysis using coherent anti-Stokes Raman scattering (CARS) and hyperspectral CARS microscopy. Eur. J. Pharm. Biopharm. 2013;85:1141-7.

31. Hartshorn CM, Lee YJ, Camp Jr CH, Liu Z, Heddleston J, Canfield N, et al. Multicomponent chemical imaging of pharmaceutical solid dosage forms with broadband CARS microscopy. Anal. Chem. 2013;85:8102-11.

32. Østergaard J, Lenke J, Sun Y, Ye F. UV Imaging for In Vitro Dissolution and Release Studies: Intial Experiences. Dissolution Technol. 2014;21:27-38

33. Tres F, Treacher K, Booth J, Hughes LP, Wren SAC, Aylott JW, et al. Real time Raman imaging to understand dissolution performance of amorphous solid dispersions. J. Controlled Release. 2014;188:53-60.

34. Gorog S. Ultraviolet-visible Spectrophotometry in Pharmaceutical Analysis: Application of UV-VIS Spectroscopy in Pharmaceutical. CRC Press; 1995.

35. Østergaard J, Meng-Lund E, Larsen SW, Larsen C, Petersson K, Lenke J, et al. Real-Time UV Imaging of Nicotine Release from Transdermal Patch. Pharm. Res. 2010;27:2614-23.

36. Jensen SS, Jensen H, Goodall DM, Østergaard J. Performance characteristics of UV imaging instrumentation for diffusion, dissolution and release testing studies. J Pharm Biomed Anal. Submitted.

37. Khan J, Rades T, Boyd B. The Precipitation Behavior of Poorly Water-Soluble Drugs with an Emphasis on the Digestion of Lipid Based Formulations. Pharm. Res. 2016;33:548-62.

38. Pouton CW. Formulation of poorly water-soluble drugs for oral administration: physicochemical and physiological issues and the lipid formulation classification system. Eur. J. Pharm. Sci. 2006;29:278-87.

39. Boetker JP, Savolainen M, Koradia V, Tian F, Rades T, Müllertz A, et al. Insights into the Early Dissolution Events of Amlodipine Using UV Imaging and Raman Spectroscopy. Mol. Pharm. 2011;8:1372-80.

40. Hulse WL, Gray J, Forbes RT. A discriminatory intrinsic dissolution study using UV area imaging analysis to gain additional insights into the dissolution behaviour of active pharmaceutical ingredients. Int. J. Pharm. 2012;434:133-9.

41. Eddleston MD, Thakuria R, Aldous BJ, Jones W. An investigation of the causes of cocrystal dissociation at high humidity. J. Pharm. Sci. 2014;103:2859-64.

42. Sun CC. Cocrystallization for successful drug delivery. Expert Opin. Drug Deliv. 2013;10:20113.

43. Thakuria R, Delori A, Jones W, Lipert MP, Roy L, Rodríguez-Hornedo N. Pharmaceutical cocrystals and poorly soluble drugs. Int. J. Pharm. 2013;453:101-25. 
44. Qiao N, Li M, Schlindwein W, Malek N, Davies A, Trappitt G. Pharmaceutical cocrystals: an overview. Int. J. Pharm. 2011;419:1-11.

45. Qiao N, Wang K, Schlindwein W, Davies A, Li M. In situ monitoring of carbamazepinenicotinamide cocrystal intrinsic dissolution behaviour. Eur. J. Pharm. Biopharm. 2013;83:415-26.

46. Li M, Qiao N, Wang K. Influence of Sodium Lauryl Sulfate and Tween 80 on CarbamazepineNicotinamide Cocrystal Solubility and Dissolution Behaviour. Pharmaceutics. 2013;5:508-24.

47. Gordon S, Naelapää K, Rantanen J, Selen A, Müllertz A, Østergaard J. Real-time dissolution behavior of furosemide in biorelevant media as determined by UV imaging. Pharm. Dev. Technol. 2013;18:1407-16.

48. Nielsen LH, Gordon S, Pajander JP, Østergaard J, Rades T, Müllertz A. Biorelevant characterisation of amorphous furosemide salt exhibits conversion to a furosemide hydrate during dissolution. Int. J. Pharm. 2013;457:14-24.

49. Niederquell A, Kuentz M. Biorelevant dissolution of poorly soluble weak acids studied by UV imaging reveals ranges of fractal-like kinetics. Int. J. Pharm. 2014;463:38-49.

50. Østergaard J, Wu JX, Naelapää K, Boetker JP, Jensen H, Rantanen J. Simultaneous UV Imaging and Raman Spectroscopy for the Measurement of Solvent-Mediated Phase Transformations During Dissolution Testing. J. Pharm. Sci. 2014;103:1149-56.

51. Boetker JP, Rantanen J, Rades T, Müllertz A, Østergaard J, Jensen H. A New Approach to Dissolution Testing by UV Imaging and Finite Element Simulations. Pharm. Res. 2013;30:1328-37.

52. Lu Y, Li M. Simultaneous Rapid Determination of the Solubility and Diffusion Coefficients of a Poorly Water-Soluble Drug Based on a Novel UV Imaging System. J. Pharm. Sci. 2016;105:1318.

53. Østergaard J, Ye F, Rantanen J, Yaghmur A, Larsen SW, Larsen C, et al. Monitoring lidocaine single - crystal dissolution by ultraviolet imaging. J. Pharm. Sci. 2011;100:3405-10.

54. Doherty C, York P. Microenvironmental pH control of drug dissolution. Int. J. Pharm. 1989;50:223-32.

55. Østergaard J, Jensen H, Larsen SW, Larsen C, Lenke J. Microenvironmental pH measurement during sodium naproxenate dissolution in acidic medium by UV/vis imaging. J. Pharm. Biomed. Anal. 2014;100:290-3.

56. Gajdošová M, Sarvašová N, Pěček D, Štěpánek F. The Use of MRI as a Technique for Hydrophilic Polymer Swelling Characterization. MRS Proc. Cambridge Univ Press; 2015. p. 11722.

57. Kulinowski P, Młynarczyk A, Jasiński K, Talik P, Gruwel ML, Tomanek B, et al. Magnetic Resonance Microscopy for Assessment of Morphological Changes in Hydrating Hydroxypropylmethylcellulose Matrix Tablets In Situ-Is it Possible to Detect Phenomena Related to Drug Dissolution Within the Hydrated Matrices? Pharm. Res. 2014;31:2383-92. 
58. Wray PS, Clarke GS, Kazarian SG. Application of FTIR spectroscopic imaging to study the effects of modifying the $\mathrm{pH}$ microenvironment on the dissolution of ibuprofen from HPMC matrices. J. Pharm. Sci. 2011;100:4745-55.

59. van der Weerd J, Kazarian SG. Release of poorly soluble drugs from HPMC tablets studied by FTIR imaging and flow-through dissolution tests. J. Pharm. Sci. 2005;94:2096-109.

60. Kazarian SG, Van der Weerd J. Simultaneous FTIR spectroscopic imaging and visible photography to monitor tablet dissolution and drug release. Pharm. Res. 2008;25:853-60.

61. Van der Weerd J, Kazarian SG. Validation of macroscopic attenuated total reflection-Fourier transform infrared imaging to study dissolution of swelling pharmaceutical tablets. Appl. Spectrosc. 2004;58:1413-9.

62. Pajander J, Baldursdottir S, Rantanen J, Østergaard J. Behaviour of HPMC compacts investigated using UV-imaging. Int. J. Pharm. 2012;427:345-53.

63. Viridén A, Wittgren B, Larsson A. Investigation of critical polymer properties for polymer release and swelling of HPMC matrix tablets. Eur. J. Pharm. Sci. 2009;36:297-309.

64. Narasimhan B. Mathematical models describing polymer dissolution: consequences for drug delivery. Adv. Drug Deliv. Rev. 2001;48:195-210.

65. Schachter DM, Xiong J, Tirol GC. Solid state NMR perspective of drug-polymer solid solutions: a model system based on poly(ethylene oxide). Int. J. Pharm. 2004;281:89-101.

66. Newman A, Nagapudi K, Wenslow R. Amorphous solid dispersions: a robust platform to address bioavailability challenges. Ther. Deliv. 2015;6:247-61.

67. Lee TWY, Boersen NA, Hui HW, Chow SF, Wan KY, Chow AHL. Delivery of poorly soluble compounds by amorphous solid dispersions. Curr. Pharm. Des. 2014;20:303-24.

68. Paudel A, Geppi M, Mooter GV den. Structural and Dynamic Properties of Amorphous Solid Dispersions: The Role of Solid-State Nuclear Magnetic Resonance Spectroscopy and Relaxometry. J. Pharm. Sci. 2014;103:2635-62.

69. Jesson G, Brisander M, Andersson P, Demirbüker M, Derand H, Lennernäs H, et al. Carbon Dioxide-Mediated Generation of Hybrid Nanoparticles for Improved Bioavailability of Protein Kinase Inhibitors. Pharm. Res. 2013;31:694-705.

70. Colombo S, Brisander M, Haglöf J, Sjövall P, Andersson P, Østergaard J, et al. Matrix effects in nilotinib formulations with $\mathrm{pH}$-responsive polymer produced by carbon dioxide-mediated precipitation. Int. J. Pharm. 2015;494:205-17.

71. Kalepu S, Manthina M, Padavala V. Oral lipid-based drug delivery systems - an overview. Acta Pharm. Sin. B. 2013;3:361-72.

72. Gautschi N, Van Hoogevest P, Kuentz M. Amorphous drug dispersions with mono- and diacyl lecithin: On molecular categorization of their feasibility and UV dissolution imaging. Int. J. Pharm. 2015;491:218-30. 
73. Gaunø MH, Vilhelmsen T, Larsen CC, Boetker JP, Wittendorff J, Rantanen J, et al. Real-time in vitro dissolution of 5-aminosalicylic acid from single ethyl cellulose coated extrudates studied by UV imaging. J. Pharm. Biomed. Anal. 2013;83:49-56.

74. Junghanns J-UAH, Muller RH. Nanocrystal technology, drug delivery and clinical applications. Int. J. Nanomedicine. 2008;3:295-309.

75. Gao L, Liu G, Ma J, Wang X, Zhou L, Li X. Drug nanocrystals: in vivo performances. J. Controlled Release. 2012;160:418-30.

76. Pawar VK, Singh Y, Meher JG, Gupta S, Chourasia MK. Engineered nanocrystal technology: in-vivo fate, targeting and applications in drug delivery. J. Control. Release Off. J. Control. Release Soc. 2014;183:51-66.

77. Sarnes A, Østergaard J, Jensen SS, Aaltonen J, Rantanen J, Hirvonen J, et al. Dissolution study of nanocrystal powders of a poorly soluble drug by UV imaging and channel flow methods. Eur. J. Pharm. Sci. 2013;50:511-9.

78. Nielsen LH, Melero A, Keller SS, Jacobsen J, Garrigues T, Rades T, et al. Polymeric microcontainers improve oral bioavailability of furosemide. Int. J. Pharm. 2016;504:98-109.

79. Chirra HD, Desai TA. Multi-Reservoir Bioadhesive Microdevices for Independent RateControlled Delivery of Multiple Drugs. Small. 2012;8:3839-46.

80. Nielsen LH, Nagstrup J, Gordon S, Keller SS, Østergaard J, Rades T, et al. pH-triggered drug release from biodegradable microwells for oral drug delivery. Biomed. Microdevices. 2015;17:1-7.

81. Shi Y, Li LC. Current advances in sustained-release systems for parenteral drug delivery. Expert Opin. Drug Deliv. 2005;2:1039-58.

82. Wang L, Liu Y, Zhang W, Chen X, Yang T, Ma G. Microspheres and microcapsules for protein delivery: strategies of drug activity retention. Curr. Pharm. Des. 2013;19:6340-52.

83. Wu F, Jin T. Polymer-Based Sustained-Release Dosage Forms for Protein Drugs, Challenges, and Recent Advances. AAPS PharmSciTech. 2008;9:1218-29.

84. Dadhaniya TM, Sharma OP, Gohel MC, Mehta PJ. Current approaches for in vitro drug release study of long acting parenteral formulations. Curr. Drug Deliv. 2015;12:256-70.

85. Seidlitz A, Weitschies W. In-vitro dissolution methods for controlled release parenterals and their applicability to drug-eluting stent testing. J. Pharm. Pharmacol. 2012;64:969-85.

86. Ye F, Yaghmur A, Jensen H, Larsen SW, Larsen C, Østergaard J. Real-time UV imaging of drug diffusion and release from Pluronic F127 hydrogels. Eur. J. Pharm. Sci. 2011;43:236-43.

87. Ye F, Larsen SW, Yaghmur A, Jensen H, Larsen C, Østergaard J. Real-time UV imaging of piroxicam diffusion and distribution from oil solutions into gels mimicking the subcutaneous matrix. Eur. J. Pharm. Sci. 2012;46:72-8. 
88. Ye F, Larsen SW, Yaghmur A, Jensen H, Larsen C, Østergaard J. Drug release into hydrogelbased subcutaneous surrogates studied by UV imaging. J. Pharm. Biomed. Anal. 2012;71:27-34.

89. Ye F, Baldursdottir S, Hvidt S, Jensen H, Larsen SW, Yaghmur A, et al. Role of Electrostatic Interactions on the Transport of Druglike Molecules in Hydrogel-Based Articular Cartilage Mimics: Implications for Drug Delivery. Mol. Pharm. 2016;13:819-28.

90. Jensen SS, Jensen H, Cornett C, Møller EH, Østergaard J. Insulin diffusion and self-association characterized by real-time UV imaging and Taylor dispersion analysis. J. Pharm. Biomed. Anal. 2014;92:203-10.

91. Jensen SS, Jensen H, Cornett C, Møller EH, Østergaard J. Real-time UV imaging identifies the role of $\mathrm{pH}$ in insulin dissolution behavior in hydrogel-based subcutaneous tissue surrogate. Eur. J. Pharm. Sci. 2015;69:26-36.

92. Jensen SS, Jensen H, Møller EH, Cornett C, Siepmann F, Siepmann J, et al. In vitro release studies of insulin from lipid implants in solution and in a hydrogel matrix mimicking the subcutis. Eur. J. Pharm. Sci. 2016;81:103-12.

93. Hoang Thi TH, Chai F, Leprêtre S, Blanchemain N, Martel B, Siepmann F, et al. Bone implants modified with cyclodextrin: Study of drug release in bulk fluid and into agarose gel. Int. J. Pharm. 2010;400:74-85.

94. Chen J-X, Guo Z, Li H-Y, Wu L, He Z-G, Hu R-F, et al. Real-time UV imaging of chloramphenicol intrinsic dissolution characteristics from ophthalmic in situ gel. Yao Xue Xue Bao. 2013;48:1156-63. 


\section{Legends to Figures}

Fig. 1 (A) Schematic representation of the commercially available Sirius SDI with Actipix ${ }^{\mathrm{TM}}$ technology UV dissolution imaging showing the key components of the instrument. (B) Schematic representation of the side-view of the UV imaging experimental setup.

Fig. 2 Schematic of the sample cells applied in UV imaging systems. Pink represents the formulation or drug substance and white represents the release/dissolution medium. (A) Flowthrough cell used for studying dissolution $(7.5 \mathrm{~mm} \times 3.0 \mathrm{~mm} \times 63.0 \mathrm{~mm}(\mathrm{H} \times \mathrm{W} \times \mathrm{L}))$. (B) Rectangular cell with one side filled with formulation, the other side with dissolution media while stirring $(7.5 \mathrm{~mm} \times 3.0 \mathrm{~mm} \times 63.0 \mathrm{~mm}(\mathrm{H} \times \mathrm{W} \times \mathrm{L}))$. $(\mathrm{C})$ Rectangular cell with one side filled with formulation, the other side with dissolution media at stagnant condition for studying diffusion or partitioning in a gel matrix $(8.0 \mathrm{~mm} \times 1.0 \mathrm{~mm} \times 38 \mathrm{~mm}(\mathrm{H} \times \mathrm{W} \times \mathrm{L}))$. (D) Formulation injected into dissolution media through syringe $(7.0 \mathrm{~mm} \times 3.0 \mathrm{~mm} \times 63 \mathrm{~mm}(\mathrm{H} \times \mathrm{W} \times \mathrm{L}))$. (E) Circular cell for studying drug release in hydrogel matrixes $(15 \mathrm{~mm}$ (id) $\times 5 \mathrm{~mm}$ ).

Fig. 3 (A) Representative images of HP55 compacts showing swelling in citrate buffer, pH 2.2 and (B) dissolution after $\mathrm{pH}$ change to $\mathrm{pH}$ 6.4. Adapted with permission from Elsevier [70]. 

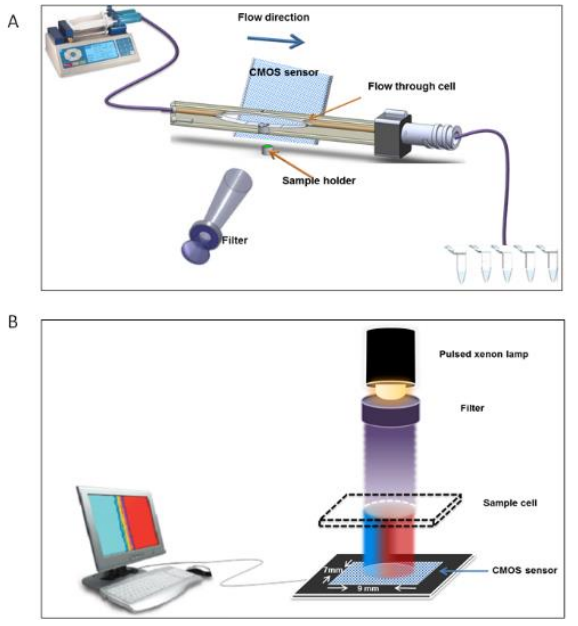

Fig. 1

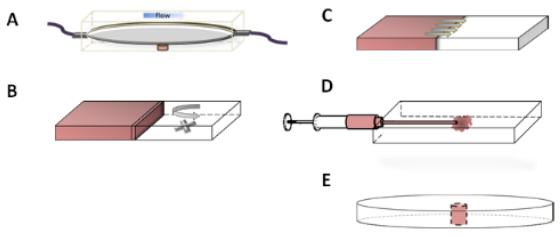

Fig. 2

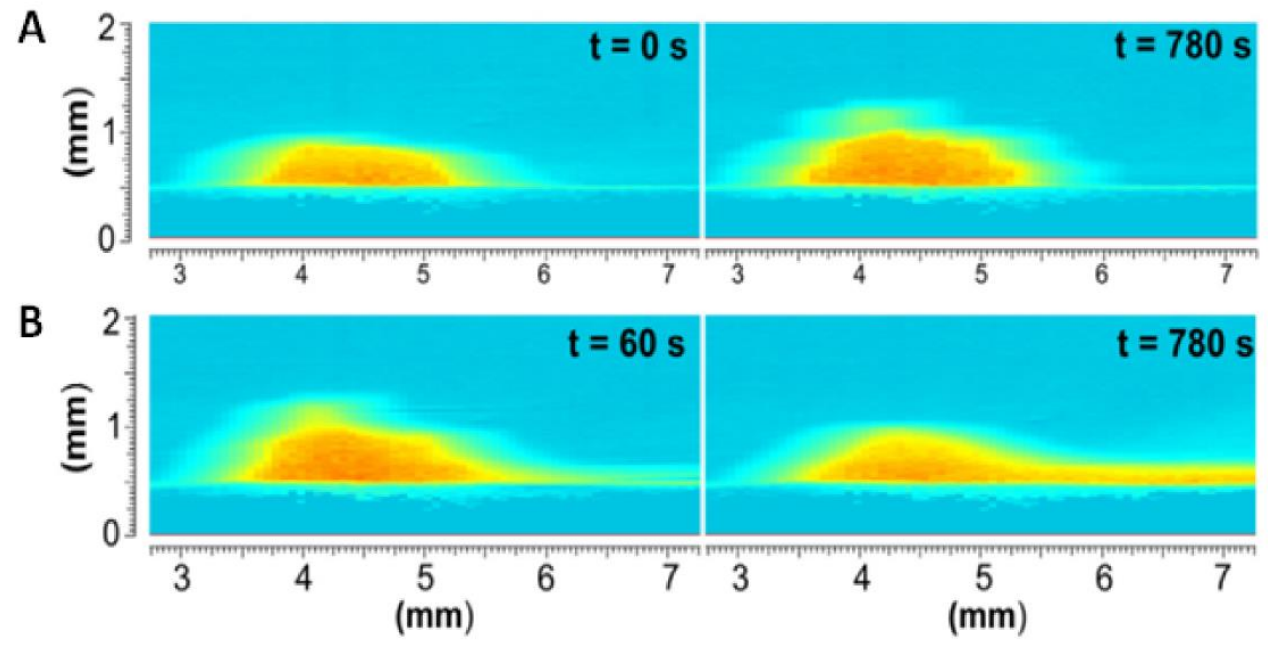

Fig. 3 